\title{
Prick, patch or blood test? A simple guide to allergy testing
}

\author{
Leelavathi Muthupalaniappen, Adawiyah Jamil \\ Muthupalaniappen L, Jamil A. Prick, patch or blood test? A simple guide to allergy testing. Malays Fam Physician. 2021;16(2);19-26. \\ https://doi.org/10.51866/rv1141
}

\section{Keywords:}

Hypersensitivity;

immunoglobulin E; skin tests;

patch test; prick test

\section{Authors:}

\section{Adawiyah Jamil \\ (Corresponding author) \\ AdvMDerm \\ Department of Medicine \\ University Kebangsaan Malaysia \\ Medical Center, Kuala Lumpur \\ Malaysia \\ Email: adawiyahjamil@ukm.edu.my}

Leelavathi Muthupalaniappen

MMed

Department of Family Medicine

University Kebangsaan Malaysia

Medical Center, Kuala Lumpur

Malaysia

\begin{abstract}
This article provides information on allergy testing and serves as a simple guide for physicians who are considering using allergy testing as a step in patient management. Basic principles of allergy testing, indications for testing, and how and when to choose a suitable allergy test are discussed. Allergy testing in general refers to evaluation of either type I or type IV hypersensitivity reactions. The type I (immediate) reaction is evaluated using the skin prick test (in vivo) or serum $\operatorname{IgE}$ (in vitro) test methods, while the type IV (delayed) reaction is determined via the skin patch test method. The allergens responsible for a specific reaction can be identified from allergy testing, and this information is useful in administering avoidance measures. Appropriate treatment of allergic reactions along with allergen avoidance ensure a successful treatment outcome and prevent future reactions.
\end{abstract}

\section{Introduction}

Allergies are described as abnormal harmful immune responses to harmless substances, or allergens. ${ }^{1}$ Both dermatologists and nondermatologists widely use allergy testing in evaluation of allergies and diseases associated with allergies. Common dilemmas concerning allergy testing include the indication for allergy testing, selection of the appropriate test, interpretation of the results and application of this information in patient management. Comprehension of the basic principles of allergy testing and the different types of tests available is helpful when considering these tests in clinical practice.

In most cases, a suspected allergen can be identified through detailed histories of symptoms and signs in relation to exposure or contact. Drawing a timeline of events is often useful to identify the suspected offending agent and develop a provisional diagnosis. Type 1 allergic reactions frequently present with cutaneous, aural, nasal, guttural, respiratory, ocular and sometimes gastrointestinal symptoms such as wheezing, breathing difficulty, shortness of breath, mouth breathing, nasal congestion, swelling and tearing of eye (red flag signs for angioedema), swelling of the lips, itching, hives, abdominal discomfort and diarrhoea.

Type IV reactions tend to present with purely cutaneous symptoms such as pruritic skin lesions. A personal or family history of atopy is essential because individuals with these histories are genetically predisposed to produce $\mathrm{IgE}$ toward certain environmental allergens. ${ }^{2}$ Patients with atopic dermatitis have a defective skin barrier that allows allergens to penetrate easily, thus causing allergic contact dermatitis. $^{3-5}$

Physical examination of patients presenting with allergic manifestations should include blood pressure, pulse rate, respiratory rate, oxygen saturation if there is tachypnoea, and rhonchi. The lips, tongue, oral mucosa and throat should be examined for angioedema, while the skin should be examined for presence of urticaria, maculopapular eruptions or vesicular lesions, and signs of atopic dermatitis. $^{6}$

Avoidance of the suspected allergen alone may bring about a favourable clinical outcome, thereby eliminating the need for allergy testing in most cases. Allergy testing may be considered in cases where withdrawal of the suspected allergen and medical management are unsatisfactory. Allergy testing should not be done as a screening for asymptomatic person or when the test result is not expected to change the patient's management outcome. ${ }^{6}$ Patients themselves or the parents of patients often request allergy testing to identify possible allergens for preventive measures; this poses a dilemma, however, because allergy testing 
without symptoms is difficult to interpret and apply clinically. For example, a positive prick test in the absence of symptoms does not indicate allergy but rather merely suggests sensitization, and allergen avoidance may not be necessary. ${ }^{7}$ Unnecessary preventive measures such as food restriction may cause anxiety among parents as well as possible malnutrition in children. ${ }^{8}$

\section{Types of allergic reactions and principles of allergy testing}

Some basic information regarding types of allergic reactions and the principles of testing are necessary to understand allergy testing better. Hypersensitivity reactions are generally classified as a type I, II, III and IV reactions.? The term 'allergy testing', in general, refers to evaluation for suspected type I or type IV hypersensitivity reactions that occur because of exogenous allergens. On the contrary, type II hypersensitivity reactions occur because of the presence of endogenous IgM or IgG antibodies, for example, in acute haemolytic transfusion reaction, rheumatic fever and pemphigus vulgaris. Type III reactions, in turn, are due to antigen-antibody complexes that result in hypersensitivity reactions, as in systemic lupus erythematosus, serum sickness, reactive arthritis, $\operatorname{IgA}$ vasculitis and nephropathy. Type II and III reactions are not evaluated via allergy testing. ${ }^{10}$

The type I reaction, also known as the immediate hypersensitivity reaction, occurs because of $\operatorname{IgE}$ activation of mast cells in the skin as well as the gastrointestinal and respiratory mucosal linings. Food (e.g. seafood), drugs, transfusion products, insect venom, and environmental or inhaled allergens such as pollen, animal dander and house dust mites are common triggers. Previous exposure to the allergen is necessary for this reaction to manifest. The first exposure produces sensitization, and subsequent exposure to the same allergen triggers a cascade of events that produces a reaction within minutes (immediate reaction) and up to 1 to 3 days afterward (late phase reaction). This reaction may present as acute urticaria, pruritus, rhinitis, conjunctivitis, bronchospasm, diarrhoea, angioedema or anaphylaxis. Type I allergic reactions are easier than type IV reactions to diagnose because the offending allergens are often identified from a detailed history of the episode and preceding events.

The type IV reaction, also known as the delayed hypersensitivity reaction, is T-cell mediated and also involves two phases. The initial sensitization phase occurs when contact is made with the allergen, which penetrates the skin. Repeated exposure to the same allergen triggers an inflammatory reaction manifesting as cutaneous lesions, usually within 12 to 48 hours of exposure, for weak allergens. This reaction commonly presents on the contact area (localized) as pruritic and erythematous papules, macules or vesicles that may spread through the systemic immune response and become generalized. This phenomenon is known as contact dermatitis with secondary generalization (or id reaction). Contact dermatitis, drug reactions (e.g. StevensJohnson syndrome), and allergic reactions to implants (e.g. joint or dental implants) are some examples of type IV reactions. ${ }^{11}$ These reactions are more complicated because the temporal relationship between allergen exposure with clinical manifestation is often not clear. Morphology of the rash is commonly indistinguishable from endogenous eczema and symptoms, such as itch or erythema, and is highly influenced by environmental factors such as heat, sweat and dust.

Contact dermatitis is further categorized as irritant contact dermatitis (ICD) and allergic contact dermatitis (ACD). ICD occurs because of direct exposure of an irritant substance (e.g. acid or alkali) on the skin or mucosal membrane without involving the immunological pathway. This reaction occurs rapidly after exposure within minutes or hours (sometimes longer), and a similar reaction occurs upon subsequent exposure to the same irritant. Skin lesions due to ICD are usually limited to the area of contact, with a welldefined margin and present with a burning sensation or pain. Pruritus is usually absent. ${ }^{12}$ In contrast, $\mathrm{ACD}$ requires prior sensitization, is triggered by the $T$ cell-mediated immunological pathway and occurs within 1 to 7 days after exposure. ${ }^{13}$ This type of dermatitis usually involves areas beyond contact and presents mainly with pruritus. Sometimes, ACD occurs after many years of repeated exposure to a weak allergen that was used in the past without any problems rather than after a recent or new contact. $^{10}$ Differentiating ICD and ACD may be difficult clinically but is nevertheless important before the patient is labelled as allergic to a substance. ${ }^{6}$ Substances causing ICD and ACD must be avoided to prevent recurrent episodes. 
Because the pathways for type I and type IV allergic reactions are different, the tests required to elicit these reactions also differ; the type I reaction is evaluated using either a skin prick test (in vivo) or a serologic test (in vitro), while the type IV reaction is evaluated using a skin patch test. ${ }^{14}$

\section{Discussion \\ Types of allergy tests \\ 1) Prick test}

The prick test works on the principle of activating $\operatorname{IgE}$ antibodies on the skin's mast cells. Allergens in the form of liquid droplets are placed on the skin surface least $2 \mathrm{~cm}$ apart on the flexor aspect of the forearm. A sterile needle or lancet is introduced into the skin through the droplet, and the needle then pushes the allergen into the dermis. A new lancet is used for every allergen to prevent contamination. An alternative method is the intradermal test, where a small amount of an allergen is injected directly into the dermis using a syringe and needle. Both techniques require a positive (histamine) and a negative (saline) control. ${ }^{6}$

The test area is examined after 15 to 20 minutes to evaluate the skin reaction. Presence of a wheal with a diameter of $3 \mathrm{~mm}$ or greater is considered as indicative of a positive reaction. ${ }^{6}$ Larger wheals indicate higher sensitivity to the allergen but not necessarily more severe allergic symptoms. The absence of wheal formation with an allergen indicates the absence of an allergy to that specific allergen; the positive control should form a wheal, while the negative control should not produce any reaction. If a wheal formation does not occur with the positive control, the patient could have inadvertently taken an antihistamine prior to the test, which causes dampening of the reaction. Results of the test would then be rendered invalid. Formation of a wheal and flare with the negative control suggests hypersensitive skin/dermographism. Cross-reactivity may occur, meaning that the sensitivity to one allergen may cause a reaction with another even if there was no previous exposure. ${ }^{6}$ The sensitivity and specificity of the prick test to aeroallergens is about $70-97 \%$, and to food allergens, the sensitivity and specificity is $30-90 \%$. $^{\text {? }}$
Indications for the prick test:, 14

- $\quad$ Suspected food allergy (e.g. to egg, peanut, wheat, fish, soy or cow's milk) that cannot be determined from food elimination or challenge.

- Poorly controlled or frequent exacerbation of allergic rhinitis, rhinosinusitis, eczema or bronchial asthma, where identifying and avoiding allergens (animal dander, pollen, cockroaches and house dust mites) may improve the condition.

- $\quad$ Suspected or previous allergy to a drug such as penicillin (only when there are limited alternative drugs which can be used for treatment).

Food allergy commonly presents as acute urticaria, angioedema or gastrointestinal symptoms. Chronic urticaria (> 6 weeks) is rarely due to an allergic reaction; hence, allergy testing is not beneficial in these cases.

\section{Precautions and contraindications for the} prick test: ${ }^{6,14}$

- Patients on treatment with oral antihistamine and antidepressants need to defer treatment before the test ( 3 days for 1 st-generation and 10 days for 2 nd-generation antihistamines) to avoid wheal dampening.

- Application of topical steroids at the test site should be stopped 2 to 3 weeks prior to testing because it may dampen the results. However, oral or inhaled steroids may be continued because use of these substances does not affect the results.

- The prick test is contraindicated in patients who experienced anaphylaxis 4 to 6 weeks prior to the test.

- The prick test in the elderly may not be accurate because skin reactivity declines with age. Serum IgE testing may be considered instead.

- It is best to defer the prick test for children below 2 years of age and pregnant women.

- There is a small chance of anaphylaxis during the test. Hence, testing should be performed where health professionals and emergency resuscitation facilities are available. 


\section{2) Serologic (serum IgE) testing}

The serologic or blood test for diagnosing allergies is based on the principle of detecting circulating $\mathrm{IgE}$ antibody levels against specific allergens in the blood. Blood assays are performed using different techniques, such as radioallergosorbent tests (RAST) or enzyme-linked immunosorbent assay, depending on equipment availability. A single allergen or a battery of allergens (between 36 to 54 or more) can be tested, and selection of the suspected allergen or allergens is shortlisted from the patient's history. Common allergens are food, plants, house dust mites, and animal dander. Sensitivity and specificity of food specific serum $\operatorname{IgE}$ vary according to the type of allergen; in general, sensitivity is high for common allergenic food like egg, peanut, milk and soy but specificity is low $(38-59 \%) .{ }^{15}$ Sensitivity to house dust mites and animal dander is $64-67 \%$, while specificity is $85-99 \%$ depending on the $\operatorname{IgE}$ threshold value. ${ }^{16}$ The cost for serum $\operatorname{IgE}$ testing depends on the number of allergens selected for testing.

\section{Indications for serum Ig E testing:}

- The skin prick test is not available or the suspected allergen is not available in skin prick test series.

- The patient has extensive dermatitis or dermographism that may cause a false positive with the skin prick test.

- The patient is unable to discontinue oral antihistamines.

- There is a history suggestive of an allergy, but skin prick testing is negative.

- There is a history of anaphylaxis to any allergen.

Post-prick test and serum IgE test counselling

Once the allergen has been identified or excluded using prick tests or serology testing, its clinical relevance must be determined. Patients must be informed and educated regarding allergens to avoid. However, rigid food avoidance, especially in children, may lead poor growth; hence, good nutrition intake must be ensured by providing a balance with alternative sources of nutrients. ${ }^{8}$ Fortunately, children tend to develop tolerance as they grow older in most cases of food allergy. ${ }^{6}$
Both prick tests and serum $\operatorname{IgE}$ testing have low positive predictive values, but their negative predictive value is high. ${ }^{17}$ This means that, even if the test does not identify specific allergens, it is useful to exclude allergens that are not responsible for the allergic reaction. For example, if the test did not cause a reaction to egg, patients need not avoid egg in their diet. However, false positive results may occur following both tests, and thus, results should be interpreted with caution and always correlated with history and physical examination. Anaphylaxis can be prevented through immediate administration of intramuscular epinephrine injection in cases where accidental exposure to a known allergen has occurred.

The advantages of the skin prick test compared to serologic testing are the lower cost and the shorter time to obtain results, while the disadvantages include the facts that fewer allergens can be tested, cooperation from young children is difficult, several essential drugs must be withheld and the procedure has a small risk of anaphylaxis.

\section{3) Patch test}

The patch test is the gold standard test used to detect type IV reaction (delayed hypersensitivity), and it has a sensitivity and specificity between 70 to $80 \% .^{18}$ Repeated exposure to allergens causes memory $\mathrm{T}$ lymphocytes to become activated and trigger a clinical reaction known as allergic contact dermatitis. Common allergens acquired through contact include fragrances, vehicles and preservatives in cosmetics, latex, plants, topical medications (e.g. topical antibiotics and corticosteroids), metals (e.g., nickel), adhesives, textiles and hair dyes. ${ }^{10}$

\section{Indications for the patch test:}

- $\quad$ Suspected contact or occupational dermatitis where the lesion is isolated in the area of contact.

- Worsening of existing atopic dermatitis despite adequate treatment.

- Recurrent dermatitis affecting the lips, face, hands, feet and perineum (areas that are frequently exposed to contact allergens). 
- Stasis eczema, discoid eczema and chronic recurrent eczema for which a definite cause cannot be established.

Patch testing includes a different series of allergens from the prick test, and there are about 35 to 80 allergens that are commercially available. The most commonly used series is the European Standard Battery, which consists of 34 allergens. The patient's own products, such as cosmetics,

toiletries, and hair products, can also be tested using the patch test. Selection of the allergen or allergens to be tested is based on history and suspicion. Allergens are placed in small aluminium or polyethylene chambers measuring 8 $\mathrm{mm}$ in diameter and $0.55 \mathrm{~mm}$ in depth, and they are adhered to the skin on the back (Figures 1 and 2). The patch is removed after 48 hours, and the first reading is documented. A second (final) reading is performed after 96 hours. A third reading on day 7 may be necessary for certain allergens, such as metals, that require longer contact on the skin before an assessment can be made. ${ }^{18}$
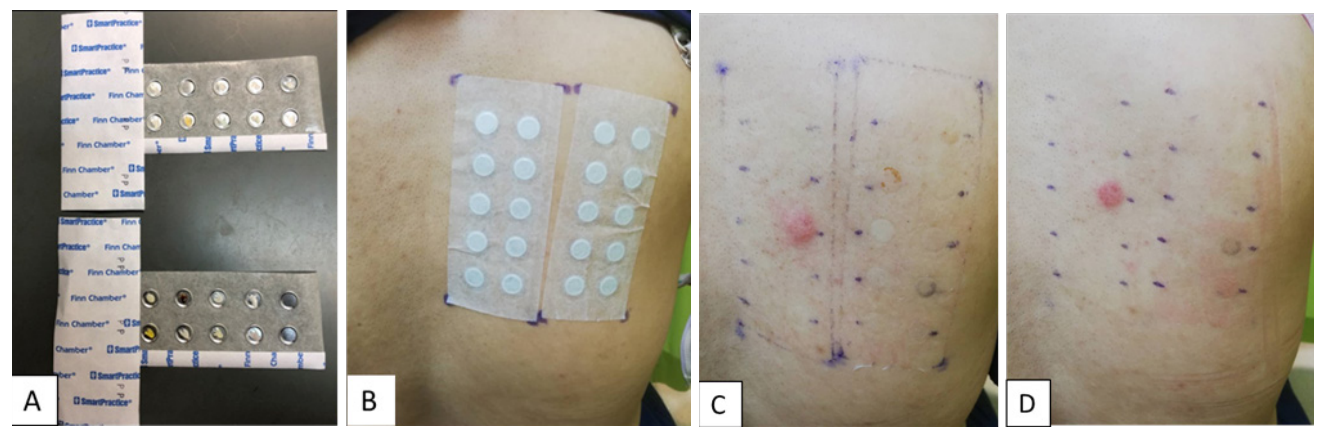

Figures A-D. A: Aluminium chambers filled with allergens. B: Allergens are patched to the upper back with hypoallergenic tape. C: Result of test on day 3 showed positive reaction to nickel. D: Result of test on day 5 showed persistence of the reaction to nickel, while negative reaction to other allergens remained.

Results are interpreted based on the degree of inflammation using criteria from the International Contact Dermatitis Research Group (ICDRG). ${ }^{10,11}$ The patch test is reported based on the presence or absence of erythema, infiltration, papules, vesicles or blisters. Reactions are documented as '+' (weak positive reaction), ' ++' (strong positive reaction) and ' +++ ' (extremely positive reaction). Some reactions are reported as 'doubtful' when the reactions do not fulfil criteria for a clear-cut positive reaction, while others produce irritation rather than allergic reactions, which differentiates ICD from ACD. ${ }^{19}$ Reporting patch test results is tedious and requires training and experience. ${ }^{10,11}$

Precautions and contraindications for the patch test: ${ }^{10,11}$

- Pre-test counselling is important because patients must refrain from bathing and exercise during the test period. Sweating and wetting of the patch causes it to dislodge, leading to test failure. Refraining from bath or ablution may not be acceptable in certain cultures; hence, informed consent must be obtained. Patients should be counselled regarding side effects such as erythema, pruritus, or hyper- or hypopigmentation at the test site. Occasionally, blisters may appear, and eczema may flare. Any skin discoloration that may occur at the test site will resolve with time.

- The test should be planned about 6 months from the time of suspected contact to avoid a flare of the existing dermatitis, especially if it is poorly controlled.

- $\quad$ Patients who are on treatment with a high daily oral steroid (> $10 \mathrm{mg}$ ), steroid injections, cyclosporin (> 2 $\mathrm{mg} / \mathrm{kg}$ ) or phototherapy, or those who have recent excessive sun exposure (within 1 week) should defer the patch test because it may affect test results. Those on low doses of oral steroids $(<10 \mathrm{mg})$ and antihistamines may proceed 
with patch testing because neither substance is known to affect the test.

- $\quad$ Topical steroid application must be avoided at the test site both 1 week before and during the test.

- The patch test should be deferred in pregnant women because immunological changes of pregnancy may affect patch results.

- A positive reaction may spread from a test site, causing a false positive result at adjacent sites. Hence, results need to be interpreted with caution.

- An irritant reaction to an allergen may cause a false positive result.

\section{Post-patch test counselling}

Patch test results should be correlated with the patient's symptoms, signs and exposure. Once the offending allergen is identified, the patient must be informed and counselled to avoid the offending allergens to prevent recurrence or persistence of symptoms. The list of substances and products that contain specific allergens can be obtained from an online contact allergen database such as http://contactallergy.com/index.html. This online information and written documentation will help to generate a list of products that contain the specific allergen that the patient needs to avoid.

The approach to select a suitable allergic test based on the presenting symptoms and signs is summarized in Figure 3.

- Acute urticaria \pm angioedema with suspected food allergens not improving with avoidance

- Recurrent or poorly controlled AR and BA despite adequate treatment

- Recurrent or poorly controlled $\mathrm{AD}$ with suspected food or aeroallergens

- Suspected allergen not available in skin prick test series

- Patient unable to discontinue oral antihistamine

- History suggestive of allergy, with negative skin prick test

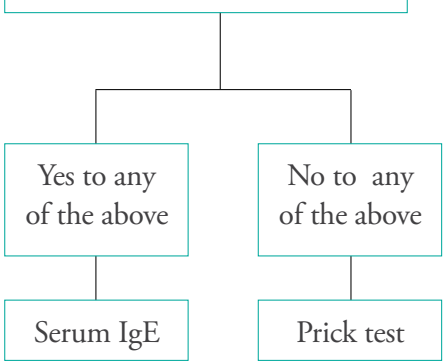

\section{Symptoms and signs}

Angioedema without urticaria

s patient on any drug?

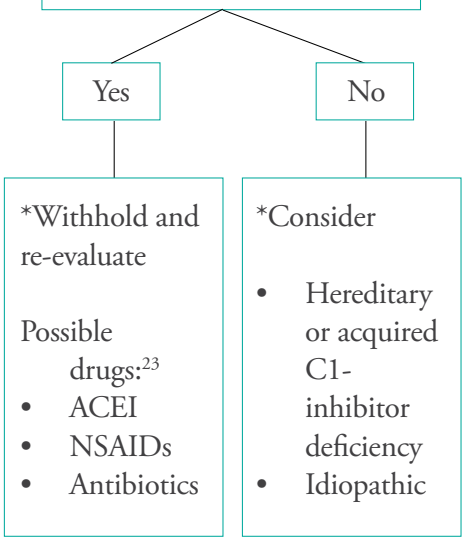

- Recurrent/poorly controlled $\mathrm{AD}$ despite adequate treatment or adult-onset $\mathrm{AD}$

- Stasis eczema, discoid eczema or chronic eczema without a clear diagnosis

- Suspected contact allergy to topical medications, cosmetics or toiletries with well-demarcated rash corresponding to area of contact

- Dermatitis affecting eyelids, head, neck, hands and/or feet

- Suspected occupational dermatitis

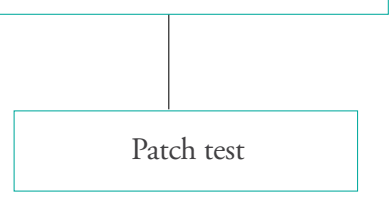

* Drug-related allergy is more complicated and follows a different algorithm. If a drug allergy or C1-inhibitor deficiency is suspected, the patient will require further evaluation by a dermatologist.

$\mathrm{AD}$ - atopic dermatitis, AR - allergic rhinitis/rhinosinusitis, BA - bronchial asthma, ACEI - angiotensin converting enzyme inhibitor

Figure 3. Approach to allergy testing 


\section{4) Other tests}

A few other tests are available for further confirmation of allergies. However, these tests are not widely available and are performed in specialized allergy centres because more advanced expertise is required for patient selection, preparation, test related materials, procedures, interpretation and application of the results.

\section{Prick to prick test}

The prick to prick test is usually performed for food allergies when commercially prepared extracts of the allergen in question are not available or a different allergenic component of the food is suspected in a negative prick test.20 Fresh fruit or vegetables are pricked, followed by immediate pricking of the skin with the same lancet. Other test procedures, reading and interpretation are similar to those of the prick test.

\section{Basophil activation test (BAT)}

Basophils, just as mast cells, will degranulate when IgE attached to highaffinity $\operatorname{IgE}$ receptors on the cell surface is cross-linked in the presence of an allergen. The basophil activation test is relatively new but has the advantage of high sensitivity and specificity. ${ }^{21}$

\section{Double-blind placebo-controlled food challenge (DBPCFC)}

This is the gold standard test in diagnosis of food allergies. The DBPCFC is guided by the outcomes of the prick test, specific $\mathrm{IgE}$ and the BAT. It involves meticulous preparation of the food and the placebo, stringent dosing schedules and multiple assessments of the clinical reactions. ${ }^{22}$

\section{Conclusion}

The prick test, the serum IgE test, and the patch test are all useful to identify common allergens responsible for clinical manifestations of allergies. The prick and serum IgE tests detect type I reactions usually related to food and aeroallergens while the patch test detects type IV reactions, which are responsible for contact dermatitis. Indication for testing must be clear because testing asymptomatic individuals is not beneficial. Clinicians must be wary of false positive or negative results, interpret the results with caution, and always match the results with a good history and physical examination. Management of allergies may be exhausting because some allergens, such as house dust mites and pollen, are constantly present in the environment, thereby rendering incessant reactions. Some allergens may be difficult to detect because they are weak and produce a reaction only after repeated exposures over months or years. Once the relevant allergens are identified and symptomatic treatment is instituted, patients must be informed and educated on allergen avoidance; this would reduce the likelihood and incidence of future reactions and ensure a satisfactory management outcome.

\section{Source of funding}

none

\section{Ethical approval}

not applicable

\section{Conflicts of interest}

both authors declare no conflicts of interest.

\section{References}

1. British Society for Immunology. Briefings and position statements. Allergy. https://www. immunology.org/policy-and-public-affairs/ briefings-and-position-statements/allergy

2. Thomsen SF. Epidemiology and natural history of atopic diseases. Eur Clin Respir J. 2015 Mar; 2: 10.3402/ecrj.v2.24642.
3. Rastogi S, Patel KR, Singam V, Silverberg JI. Allergic contact dermatitis to personal care products and topical medications in adults with atopic dermatitis. J Am Acad Dermatol. 2018 Dec; 79(6): 1028-1033.

4. de Jongh CM, Khrenova L, Verberk MM, et al. Loss-of-function polymorphisms in the filaggrin gene are associated with an increased susceptibility to chronic irritant contact dermatitis: a case-control study. Br J Dermatol. 2008 Sep; 159(3): 621-627.
5. Hamann CR, Hamann D, Egeberg A, et al Association between atopic dermatitis and contact sensitization: A systematic review and meta-analysis. J Am Acad Dermatol. 2017 Jul; 77(1): 70-78.

6. Best Practice Advocacy Centre New Zealand. Appropriate use of allergy testing in primary care. Best Tests; 2011. https://bpac.org.nz/ BT/2011/December/allergy-testing.aspx 
7. Heinzerling L, Mari A, Bergmann KC, et al. The skin prick test - European standards. Clin Transl Allergy. 2013 Feb; 3(1): 3.

8. Eichenfield LF, Tom WL, Berger TG, et al. Guidelines of care for the management of atopic dermatitis: Section 2. Management and treatment of atopic dermatitis with topical therapies. J Am Acad Dermatol. 2014 Jul; 71(1): 116-132.

9. Lundgren $\mathrm{AD}$. Allergy testing in dermatology and beyond. Cutis. 2018 Nov; 102(5): E16-E19.

10. Lazzarini R, Duarte I, Lindmayer FA. Patch tests. An Bras Dermatol. 2013 Nov; 88(6): 879888 .

11. Nosbaum A, Vocanson M, Rozieres A, et al. Allergic and irritant contact dermatitis. Eur J Dermatol. 2009 Jul; 19(4): 325-332.

12. Usatine R, Riojas M. Diagnosis and management of contact dermatitis. Am Fam Physician. 2010 Aug; 82(3): 249-255.

13. Chang KL, Guarderas JC. Allergy testing: Common questions and answers. Am Fam Physician. 2018 Jul; 98(1): 34-39.
14. Li JT. Allergy testing. Am Fam Physician. 2002 Aug; 66(4): 621-624.

15. Anvari S, Miller J, Yeh CY, et al. IgE-mediated food allergy. Clin Rev Allergy Immunol. 2019 Oct; 57(2): 244-260.

16. Hong SD, Ryu G, Seo MY, et al. Optimal cutoff values of allergen-specific immunoglobulin $\mathrm{E}$ to house dust mites and animal dander based on skin-prick test results: Analysis in 16,209 patients with allergic rhinitis. Am J Rhinol Allergy. 2018 Jan; 32(1): 23-26.

17. Sampson HA. Utility of food-specific IgE concentrations in predicting symptomatic food allergy. J Allergy Clin Immunol. 2001 Jun; 107(5): 891-896.

18. Johnston GA, Exton LS, Mohd Mustapa MF, et al. British Association of Dermatologists' guidelines for the management of contact dermatitis 2017. Br J Dermatol. 2017 Feb; 176(2): 317-329.

19. Fregert S. Manual of Contact Dermatitis. 2nd Edition. On behalf of the International Contact Dermatitis Research Group and the North American Contact Dermatitis Group. Copenhagen: Munksgaard Publishers, 1981.
20. Gomes-Belo J, Hannachi F, Swan K, et al. Advances in food allergy diagnosis. Curr Pediatr Rev. 2018; 14(3): 139-149.

21. Hoffmann HJ, Santos AF, Mayorga C, et al. The clinical utility of basophil activation testing in diagnosis and monitoring of allergic disease. Allergy. 2015 Nov; 70(11): 1393-1405.

22. Sampson HA, Gerth van Wijk R, BindslevJensen C, et al. Standardizing double-blind, placebo-controlled oral food challenges: American Academy of Allergy, Asthma \& Immunology-European Academy of Allergy and Clinical Immunology PRACTALL consensus report. J Allergy Clin Immunol. 2012 Dec; 130(6): 1260-1274.

23. Leeyaphan C, Kulthanan K, Jongjarearnprasert $\mathrm{K}$, et al. Drug-induced angioedema without urticaria: prevalence and clinical features. J Eur Acad Dermatol Venereol. 2010 Jun; 24(6): 685691. 